\title{
Recent Progress in Ultrafast Laser Nanostructuring: From Two-Plasmon Decay to Longitudinal Field Puzzle
}

\author{
P. G. Kazansky ${ }^{1}$, M. Beresna ${ }^{1}$, M. Gecevičius ${ }^{1}$, J. Zhang ${ }^{1}$, A. Patel ${ }^{1}$, R. Drevinskas ${ }^{1}$ and A. G. Kazanskii ${ }^{2}$ \\ ${ }^{I}$ Optoelectronics Research Centre, University of Southampton, SO17 1BJ, United Kingdom \\ ${ }^{2}$ Physics Department, M.V. Lomonosov Moscow State University, Moscow, Russia \\ pgk@orc.soton.ac.uk
}

\begin{abstract}
Correlation between 3/2 harmonic, two-plasmon decay and ultrafast laser induced nanogratings is observed. Paradoxically, no crystallization of amorphous Si by longitudinal field produced by $\mathrm{S}$-waveplate is observed, despite strongest intensity.

OCIS codes (140.3390) Laser materials processing, (320.7120) Ultrafast phenomena
\end{abstract}

Modification of transparent materials with ultrafast lasers has attracted considerable interest due its new science and a wide range of applications including laser surgery, integrated optics, microfluidics, optical data storage and 3D micro- and nano-structuring.

Three different types of material modifications are observed with ultrafast laser irradiation in the bulk of a transparent material, e.g. in silica glass with increase of fluence: an isotropic refractive index change; a form birefringence associated with self-assembled nanogratings with features smaller than $20 \mathrm{~nm}$ [1,2]; and a void. Considerable efforts have been carried out to clarify the mechanisms of material modifications, which are still not fully understood.

It was suggested that two-plasmon parametric decay could be involved in the mechanism of nanogratings formation [3]. A signature of two-plasmon decay is the generation of the $3 / 2$ harmonic, which previously was reliably reported only in laser plasma experiments at high intensities [4]. In order to prove two-plasmon mechanism, the observation of the $3 / 2$ harmonic at the regimes of nanograting formation is required. Here we present explicit evidence of two-plasmon decay at the laser pulse energies close to ultrafast laser nanostructuring. In addition the tight control of nanostructures' parameters is demonstrated implementing elements with unique optical properties, in particular Super-Structured-waveplates (S-waveplates) for uniform to radial and azimuthal polarization and vortex conversion, which can be used in material processing, microscopy, optical trapping and manipulation $[5,6]$. We demonstrate here that thin film of amorphous silicon exhibits anomalous polarization selective absorption for focused radially polarized beams with strong longitudinal electric field component.

The experiments were performed with femtosecond laser system Pharos (Light Conversion Ltd.) based on a directly diode pumped $\mathrm{Yb}: \mathrm{KGW}$ crystal, operating at $1030 \mathrm{~nm}$ and delivering pulses of $270 \mathrm{fs}$ at $200 \mathrm{kHz}$

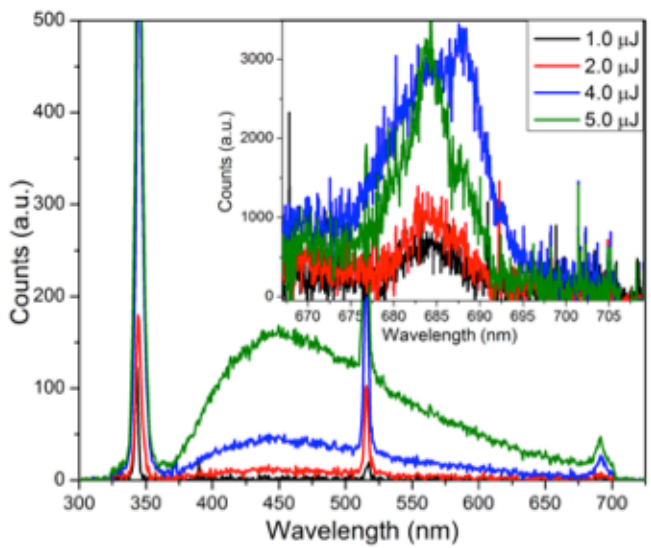

Fig. 1 Spectrum of harmonics for varying pulse energies. White light emission and the double peak structure for $3 \omega / 2$ are observed as pulse energy is increased.

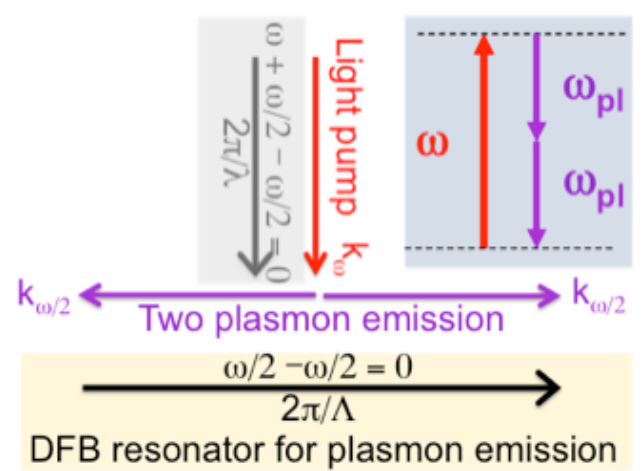

Fig.2 Diagram of nanostructure formation mechanism. Incoming photon interferes with the two plasmons generated from decay to produce nanograting of the period $2 \pi / \Lambda$. Nanograting acts as DFB resonator for plasmon emission.

repetition rate. The laser beam was focused into the bulk of the silica sample via a $\times 10(\mathrm{NA}=0.16)$ aspheric lens. The signal was collected into a fibre by collimating the transmitted light along the line of incidence with a $\times 10$ $(\mathrm{NA}=0.16)$ aspheric lens. The spectrum was analysed with an Andor Shamrock SR-500i imaging spectrometer. Within the measured spectrum, $2^{\text {nd }}, 3^{\text {rd }}$ and $3 / 2$ harmonics are observed with white light emission being more evident as pulse energy increases (Fig. 1). All three harmonics increase with pulse energy and evolve on the time scale of several minutes. All three harmonics are polarized in the direction of the pump beam's polarization. 
The observed emission of the three halves harmonic clarifies a long-standing issue on electron concentration values in ultrafast laser material modification regimes. Since the two plasmon decay is possible for the electron concentration $n_{e}=n_{c r} / 4$, where $n_{c r}$ is the critical plasma density, we could state electron concentration is at least $4 \times 10^{20} \mathrm{~cm}^{-3}$. The experimental observations provide evidence that plasmons are being excited in the bulk of the material. We suggest that the nanograting formation along the light polarization involves the interference between the two plasmons generated from the parametric decay process (Fig. 2). The nanograting produces distributed feedback (DFB) reducing the threshold of two-plasmon emission.

In the experiments on application of directly written nanostructures the radial polarization was produced by inserting a nanostructured S-waveplate [6] into the path of linearly polarized laser beam just before the objective. The ring-shapped beam was generated by modulating beam's phase with a liquid crystal SLM.

The tightly focused ring-shaped radially polarized beam should create a strong longitudinal electric field, whose intensity is twice as high as the transverse component with a long depth of focus $(\sim 24 \lambda)$ (Fig. 3). The presence of longitudinal component was confirmed by SHG in z-cut lithium niobate wafer. The SH was generated by focusing annular radially polarized beam with a 1.2 NA water immersion objective. We observed twice higher SH power for the radially polarized annular beam compared with the azimuthally polarized corresponding to the contribution of the pump longitudinal field towards the transverse field of the SH. In the near field the SH formed a radially polarized ring (Fig. 4). In the far field SH signal was in the shape of three-lobes for radial and azimuthal polarizations indicating spatially variant phase of the beam. Moreover, the pattern of the second harmonic was defined by the orientation of lithium niobate.

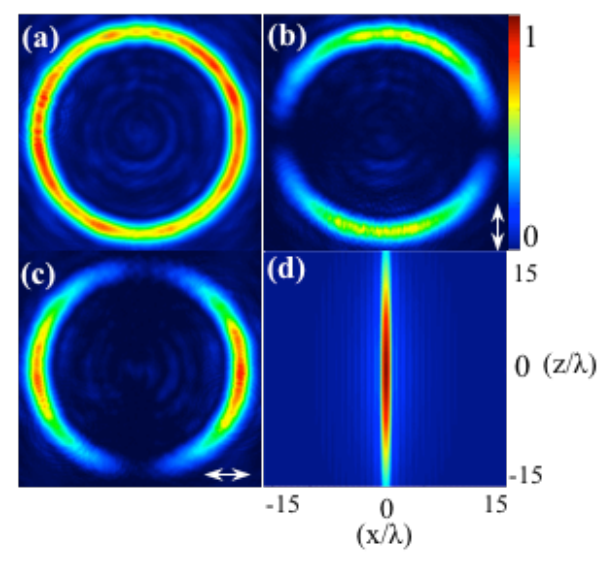

Fig. 3 Measured intensity distributions for radially polarized ring-shaped beam: (a) in the cross-section of the beam; (b and c) imaged through linear polarizer; (d) in the plane of propagation when beam is focused with a 1.2 NA objective.
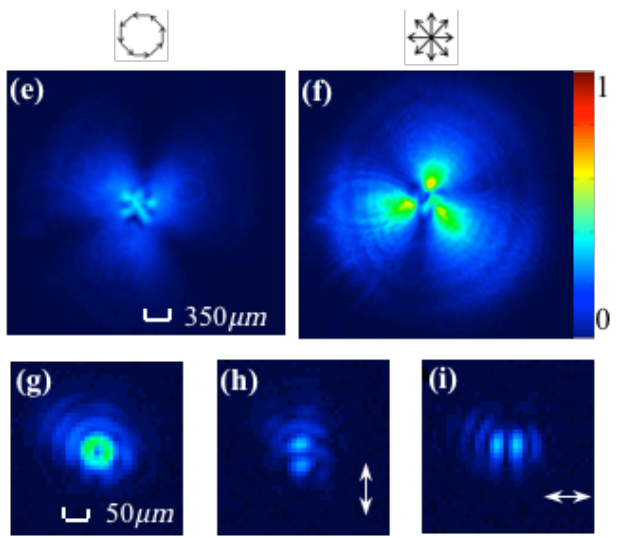

Fig. 4 Intensity distributions of second harmonic generated in lithium niobate: In the far field by radially (e) and azimuthally (f) polarized pump; (g) in the near field by radially polarized pump; (h) and (i) imaged through linear polarizer.

The ring-shaped radially polarized beam was used to modify $300 \mathrm{~nm}$ thick hydrogenated amorphous silicon films [7]. With annular beam the difference between radial and azimuthal polarizations was very strong. The threshold for permanent modification threshold was more than $40 \%$ lower for radial polarization than for azimuthal. This indicates larger fluence of the radially polarized annular beam, which should be related to the enhancement of longitudinal polarization. However, in both cases the ring shape of laser printed dots was observed. The middle of the structure with the strongest longitudinal field was not modified. This contradicts common notion that strength of modification should be dependent on the fluence of the laser beam. We speculate that the curious effect could be explained by the coupling of the longitudinal electric field of light with the surface waves, which redistribute energy over large area.

\section{References}

[1] Y. Shimotsuma et al., "Self-organized nanogratings in glass irradiated by ultrashort light pulses,” Phys. Rev. Lett. 91, 247405 (2003) .

[2] V. Bhardwaj et al, "Optically produced arrays of planar nanostructures inside fused silica," Phys. Rev. Lett. 96, 057404 (2006).

[3] P. G. Kazansky et al., "Self-assembled nanostructures and two-plasmon decay in femtosecond processing of transparent materials," in Conference on Lasers and Electro-Optics/Quantum Electronics, (OSA, 2007), paper CThJ3.

[4] W. L. Kruer, "The physics of laser plasma interactions," (Westview Press, 2001).

[5] R. Dorn et al, "Sharper focus for a radially polarized light beam," Phys. Rev. Lett. 91, 233901 (2003).

[6] M. Beresna et al., "Radially polarized optical vortex converter created by femtosecond laser nanostructuring of glass," Appl. Phys. Lett. 98, 201101 (2011); www.altechna.com/news_and_events.php

[7] A. V. Emelyanov et al., "Visible luminescence from hydrogenated amorphous silicon modified by femtosecond laser irradiation," Appl. Phys. Lett. 101, 081902 (2012). 